\title{
Do we know the actual magnetopause position for typical solar wind conditions?
}

A. A. Samsonov, ${ }^{1}$ E. Gordeev, ${ }^{1}$ N. A. Tsyganenko, ${ }^{1}$ J. Šafránková, ${ }^{2}$ Z.

Němeček, ${ }^{2}$ J. Simunek, ${ }^{3}$ D. G. Sibeck, ${ }^{4}$ G. Tóth, ${ }^{5}$ V. G. Merkin, ${ }^{6}$ J. Raeder ${ }^{7}$

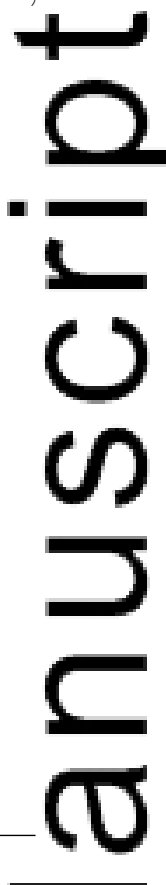

Correspongry author: A. A. Samsonov, St.Petersburg State University, 7/9 Universitetskaya nab., St. Petersburg, 199034 Russia, (a.samsonov@spbu.ru)

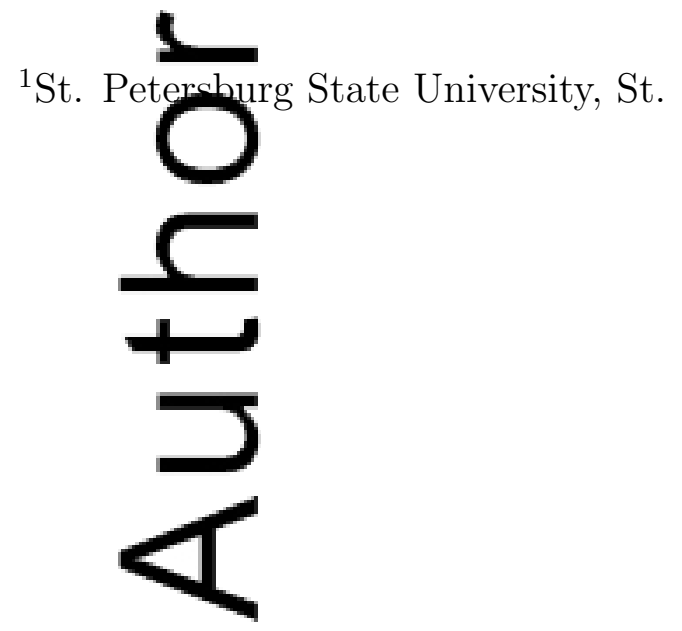

This is the author manuscript accepted for publication and has undergone full peer review but has not been through the copyediting, typesetting, pagination and proofreading process, which may lead to differences between this version and the Version of Record. Please cite this article

D RaAdEi: T10.1002/2016JA02247June 29, 2016, 9:56am

D R A F T

This article is protected by copyright. All rights reserved. 
3 Abstract. We compare predicted magnetopause positions at the subso-

4 lar point and four reference points in the terminator plane obtained from sev-

${ }_{5}$ eral empirical and numerical MHD models. Empirical models using various

6 sets of magnetopause crossings and making different assumptions about the

7 magneto predict significantly different magnetopause positions

s (with a sctter $\geq 1 R_{E}$ ) even at the subsolar point. Axisymmetric magne-

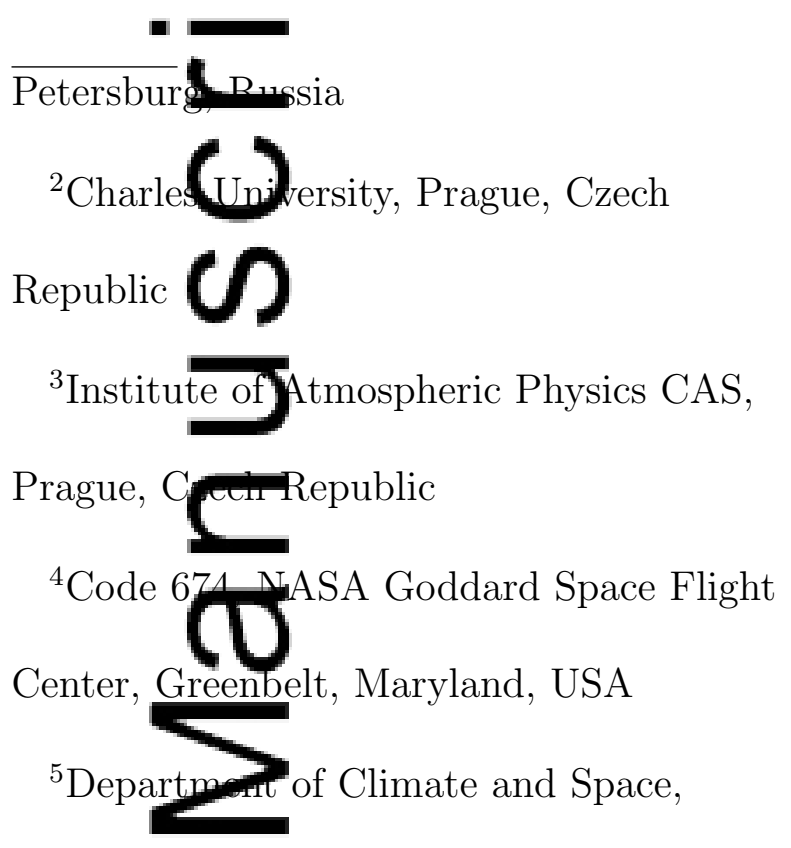

University of Michigan, Ann Arbor,

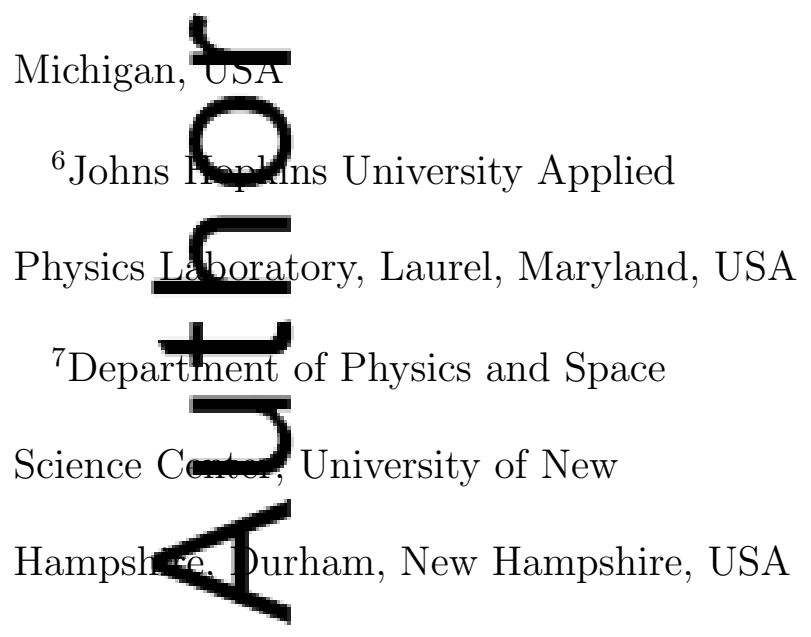


9 topause models cannot reproduce the cusp indentations or the changes re-

10 lated to the dipole tilt effect and most of them predict the magnetopause closer

11 to the Earth than non-axisymmetric models for typical solar wind conditions

${ }_{12}$ and zero tilt angle. Predictions of two global non-axisymmetric models [ Lin

${ }_{13}$ et al., 201.

${ }_{14}$ additionalveri cation. MHD models often predict the magnetopause closer

15 to the Earth than the non-axisymmetric empirical models, but the predic-

16 tions of MHD simulations may need corrections for the ring current effect

17 and decreasesof the solar wind pressure that occur in the foreshock. Com-

${ }_{18}$ paring MHD models in which the ring current magnetic field is taken into

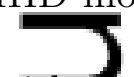

19 account wirthe empirical Lin et al. model, we find that the differences in

20 the referenosint positions predicted by these models are relatively small

${ }_{21}$ for $B_{z}=$ Therefore we assume that these predictions indicate the ac-

22 tual magrepause position, but future investigations are still needed.

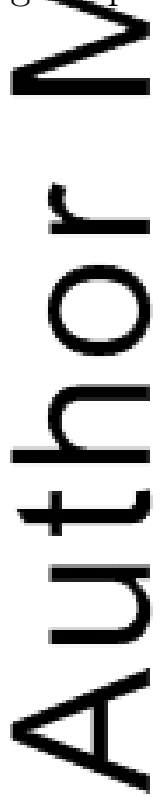




\section{Introduction}

The magnetopause is the boundary between the Earth's and interplanetary magnetic

${ }_{24}$ fields. Space weather studies require better predictions for the magnetopause shape and

${ }_{25}$ position under different solar wind conditions. The magnetopause position can be roughly

${ }_{26}$ determine 1 me pressure balance between the dynamic pressure of the supersonic

${ }_{27}$ solar wind the magnetic pressure of the Earth's dipole [e.g., Chapman and Ferraro, ${ }_{28}$ 1931; Zhigulev and Romishevskii, 1959; Beard, 1960; Spreiter and Briggs, 1962; Mead and Beard, 196lson, 1969]. This method is relatively simple, but inaccurate. First, the so total pres exen at the subsolar magnetopause is not exactly equal to the solar wind ${ }_{31}$ dynamic press $)$ re [e.g., Spreiter et al., 1966; Samsonov et al., 2012]. Second, the total 32 magnetos heric magnetic field is a superposition of magnetic fields from several current 33 systems a dipole field [e.g., Tsyganenko and Andreeva, 2015]. Later, Sotirelis and ${ }_{34}$ Meng [1009] developed a magnetopause model using the Newtonian approximation to 35 calculate external magnetosheath pressure and the T96 [Tsyganenko, 1995, 1996] ${ }_{36}$ magnetic field model to calculate the internal magnetospheric pressure, using a series of 37 numerical iterations.

However st of our knowledge about the magnetopause position comes from empirical models baden a large number of spacecraft crossings. Since Fairfield [1971], more 40 than 15 empirical magnetopause models have been developed (14 of them mentioned ${ }_{41}$ in Suvoro Dmitriev [2015]) which define the magnetopause using different sets of 42 observa 1 However, with only several exceptions [Dmitriev and Suvorova, 2000; Wang 43 et al., 2013; Shukhtina and Gordeev, 2015], all the empirical models made some a priori 
${ }_{44}$ assumptions about the magnetopause shape. For example, the well-known Shue et al.

45 [1998] model assumed the functional form

$$
R=R_{x}\left(\frac{2}{1+\cos \theta}\right)^{\alpha}
$$

${ }_{47}$ for the magnetopause, where $R$ is the radial distance, $R_{x}$ is the position of the subsolar

${ }_{48}$ point, and the solar zenith angle. This assumption may lead to significant errors in

49 some regien particular in the cusps where the magnetopause lies closer to the Earth so and the shape becomes non-axisymmetric [Boardsen et al., 2000]. Recent magnetopause ${ }_{51}$ models [Bownen et al., 2000; Lin et al., 2010; Wang et al., 2013] reproduce, at least ${ }_{52}$ qualitative cusp indentation, but both the Boardsen et al. [2000] and Lin et al. ${ }_{53}$ [2010] models re also based on assumed functional forms. The Wang et al. [2013] model ${ }_{54}$ uses the S

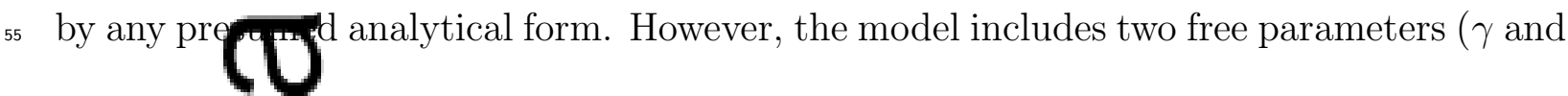
${ }_{56} C$ ) which determine the fitting procedure. The authors chose these parameters making 57 implicit amptions about most probable (rather smooth) magnetopause shape.

${ }_{58}$ Alternatively, the magnetopause shape and position can be determined using results 59 from globalMHD simulations [e.g., Elsen and Winglee, 1997; Garcia and Hughes, 2007; Lu

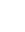

so et al., 2011. Ontrary to empirical models, the pressure balance condition in this approach ${ }_{61}$ is satisfiedterery point, and the magnetopause shape is always non-axisymmetric. But 62 the global MHD models do not include properly all magnetospheric current systems, ${ }_{63}$ in particume ring current, therefore the magnetopause position derived from MHD ${ }_{64}$ solution also be inaccurate. In this paper, we discuss these and other factors not ${ }_{65}$ considered by MHD models which may influence their predictions. 
${ }_{66}$ Recently Gordeev et al. [2015] suggested a set of benchmarks for verifying global MHD

${ }_{67}$ codes. In particular, one of the key parameters in their tests was the magnetopause

${ }_{68}$ position at the subsolar point $(y=z=0)$ and $x=0$ and $x=-15 R_{E}$ planes. They

69 compared the MHD predictions with results from the Shue et al. [1998] model at the

70 subsolar and with the Lin et al. [2010] model at other points. Gordeev et al.

${ }_{71}$ [2015] con ludd that the MHD predictions correlate well with results from the empirical

72 models in general, but sometimes underestimate or overestimate distances predicted by

73 the empirigal models. But they only briefly mentioned concerns about the accuracy of the

74 empirical magnetopause models themselves. Is it really true that the empirical models क

75 are more accurate than the MHD models and which of the empirical models is better?

${ }_{76}$ Our purpose now is to compare predictions of several empirical and MHD models for

77 typical so conditions. We are looking for systematic differences between axisym78 metric an 10 -axisymmetric empirical and MHD models at reference points and will 79 suggest foranations for these differences. We do not specifically intend to estimate the

so quality models, however we can show that predictions of some models can dif-

${ }_{81}$ fer significantly from those of the majority. We investigate ways of improving the MHD

82 models, in paricular by adding the magnetic field created by the ring current. We discuss

83 the role of the_Earth's magnetic dipole tilt.

${ }_{84}$ The magnetopause shape and position depend on the solar wind conditions and the ${ }_{85}$ Earth's dipilt angle, but most empirical models average magnetopause positions for ${ }_{86}$ different conditions using only several input parameters (usually the solar wind dynamic ${ }_{87}$ pressure Pand interplanetary magnetic field $B_{z}$ ). Therefore we prefer to compare ${ }_{88}$ results from models for idealized stationary solar wind conditions rather than study some 
s9 particular events with arbitrary pre-conditions when the magnetopause shape and size may be nonstationary and significantly differ from the average. We use typical solar wind

${ }_{91}$ conditions (see below) for which, we believe, the empirical models are most reliable.

\section{Empirical and numerical models}

\subsection{Empinical magnetopause models}

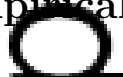

Table 1 presents a list of seven empirical and one analytical magnetopause models.

${ }_{93}$ The Petrind Russell [1996], Kuznetsov and Suvorova [1998], and Shue et al. [1998] ${ }_{94}$ models (a bre iated below as PR96, KS98, and S98 respectively) are axisymmetric, but ${ }_{95}$ use differ al alytical expressions and differ in their predictions. The analytical model 96 of Pudovkin e al. [1998] (P98) was developed from the pressure balance condition at ${ }_{97}$ the subso porint $R_{x}$. The P98 model uses both the well-known dependence $R_{x} \sim$

${ }_{98} P_{d y n}^{-1 / 6}[$ Mead_and Beard, 1964] and some assumptions about southward interplanetary 99 magnetic fiela (IMF) penetration into the magnetosphere resulting from magnetopause reconnection Boardsen et al. [2000] (B00) presented empirical models both for the highlatitude magnetopause near and behind the cusps and for the nose magnetopause. The nose magrouspe model used 290 magnetopause crossings which satisfied the criteria: ${ }_{103}$ latitude b $\mathrm{n}-81^{\circ}$ and $81^{\circ}$, and magnetic local time from 9 to 15 . Contrary to the previdus models noted above, these models consider the dipole tilt as one of input 
${ }_{110}$ Wang et al. [2013] model (W13) uses the largest database, containing 15,089 magne${ }_{111}$ topause crossings. The model has no predetermined analytical form, and consequently 112 its results for any given condition cannot be reproduced without full access to the model.

${ }_{113}$ Shukhtina and Gordeev [2015] (SG15) developed a model to determine the magnetopause ${ }_{114}$ position terminator plane in the high-latitude regions as a function of $P_{d y n}, B_{z}$ and ${ }_{115} \Psi$.

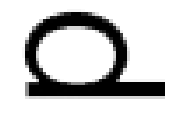

\subsection{Glowal MHD models}

We sim(late the interaction between the solar wind and magnetosphere using the ${ }_{117}$ Space We et Modeling Framework (SWMF) [Tóth et al., 2005, 2012], the SWMF ${ }_{118}$ coupled wrut Comprehensive Ring Current Model (CRCM) [Glocer et al., 2013], 119 the Lyon-Fedder-Mobarry magnetosphere-ionosphere model (LFM-MIX) [Lyon et al., 120 2004; Merkin and Lyon, 2010], and the Open Geospace General Circulation Model

122 ing Center p://ccmc.gsfc.nasa.gov). The resolution of the block-adaptive Cartesian ${ }_{123}$ grid near the magnetopause in the equatorial and terminator planes in the SWMF code ${ }_{124}$ is 0.125 The Cartesian grid resolution in the OpenGGCM code is similar to the 
${ }_{146} \quad V_{y}=V_{z}$

2013; Meng et al., 2013]. In particular, the CRCM simulates the evolution of an inner magnetospheric plasma distribution that conserves the first two adiabatic invariants. The plasma pressure obtained from the CRCM simulations modifies the pressure in the MHD code. This modification self-consistently changes other MHD parameters including the

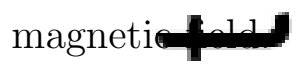

The lou altit de boundary of global MHD codes is located at a radial distance of $R \simeq$ $2-3 R_{E}$. This boundary is usually a non-penetrable sphere. The density in the SWMF 1

runs is set $28 \mathrm{~cm}^{-3}$, and in the OpenGGCM runs to $3 \mathrm{~cm}^{-3}$. In the LFM runs, the radial (normal to the boundary) gradient of the density is equal to zero. Xi et al. क

[2015] compared the low-altitude boundary conditions for several global MHD models 3

and demonstrated that these conditions may influence the accuracy of solutions. The ionospher conductances are set to constants in the runs presented below, with Pedersen conductan $\mathrm{C}=5 \mathrm{~S}$ and Hall conductance $\Sigma_{H}=0$.

We fix solar wind parameters at the outer boundary: $N=5 \mathrm{~cm}^{-3}, V_{x}=-400 \mathrm{~km} / \mathrm{s}$, $V_{y}=V_{z}$ dynamic pressure is $\left.1.34 \mathrm{nPa}\right), T=2 \times 10^{5} \mathrm{~K}, B_{y}=-B_{x}=3.5 \mathrm{nT}$ and take different $B_{z}$. We study three stationary cases with $B_{z}=0,+3,-3$ nT referred to henceforthas uns $B z 0, B z+$ and $B z-$. The dipole tilt in these three runs is set equal to zero, but we separately describe a special case with a non-zero dipole tilt angle. We usually run the codes during 3 hours with steady solar wind conditions and check that the magnetop positions at the reference points (see below) do not change during the last hour of simulations. In some MHD models, the reference point positions (in particular, along the vis) may vary in time [see also Merkin et al., 2013], and in this case we take averages over the last 30 minutes. 


\subsection{SPBU15 MHD model}

We have modified the local numerical anisotropic MHD model previously described by Samsonov et al. [2007]; Samsonov et al. [2012]. The previous code used spherical coordinates and was developed only for the dayside magnetosheath, while the new code solves single-flum equations in Cartesian coordinates for the entire magnetosphere including th Eath's dipole field as explained by Tanaka [1994]; Gombosi et al. [2002]. We apply the equations in the conservative form (in particular, calculating time variations of the total erers rather than of the thermal pressure) and maintain the $\nabla \cdot \mathbf{B}=0$ constraint using the proiection scheme, i.e. solving Poisson's equation and correcting B after a few (3)

time steps Brackbill and Barnes, 1980]. Below we will refer to this code using the working (2)

name SPBOrJ. We performed simulations using both the isotropic and anisotropic MHD codes (thenicotropic code calculates two thermal pressure components, $p_{\perp}$ and $p_{\|}$, perpendicula_a parallel to magnetic field instead of only one isotropic component $p$ ), but present the isotropic MHD results in this paper. With the given spatial resolution, we foun minnificant differences in the reference magnetopause point positions (see below) obtained by the isotropic and anisotropic codes.

The out -30 andaries of the computational domain are located at $x=-30$ $\mathrm{R}_{E}$ and at $u_{\mathbf{z}} z= \pm 40 \mathrm{R}_{E}$. The numerical grid is uniform in the whole region with a resolution of $0.5 \times 0.5 \times 0.5 \mathrm{R}_{E}^{3}$. Near the Earth (at radial distances $R \leq 5 \mathrm{R}_{E}$ where 1

the inner dary is usually located), the conditions $\mathbf{V}=0$ and $\mathbf{B}_{\mathbf{1}}=0$ (where $\mathbf{V}$ is the flow velocity and $\mathbf{B}_{\mathbf{1}}$ is the external magnetic field) are applied. The density at the inner bounc equals the solar wind density, while the thermal pressure is ten times higher than the solar wind thermal pressure. Although this model cannot reproduce the 


\section{Resưtes}

\subsection{Magnet pause shape in empirical and MHD models}


${ }_{198}$ cia and Hughes, 2007], or tracing solar wind plasma streamlines [Palmroth et al., 2003].

199 Magnetopause positions determined by the different methods may not coincide, especially 200 away from the subsolar region.

${ }_{201}$ Using strict magnetopause criteria is essential for automatic methods, but we can check ${ }_{202}$ every re eye in case studies. In this study, we identify the magnetopause as the ${ }_{203}$ peak in thele tric current density. This simple method fails to find the subsolar magne${ }_{204}$ topause in purely northward IMF cases, but gives reasonable results in most other cases. ${ }_{205}$ In empirica ndels, the magnetopause is primarily determined by the magnetic field ro${ }_{206}$ tation. Figure 1 shows the electric current density obtained by the SWMF model in the ${ }_{207}$ run $B z 0$. Local maxima of electric currents indicate both the magnetopause and bow ${ }_{208}$ shock postrons, but the maximum at the dayside magnetopause is usually higher than 209 that at thew shock. The boundary between open and closed magnetic field lines nearly ${ }_{210}$ coincides il the electric current maximum in the low-latitude region sunward of the ${ }_{211}$ termina $x=0$ ) plane. In the meridional plane, two high-latitude indentations on the magnet urface are formed above the northern and southern cusps. In the termina${ }_{213}$ tor plane, the magnetopause is deformed so that the cusp indentations are slightly rotated ${ }_{214}$ clockwise ifog fing from the Sun in accordance with the IMF orientation along the Parker ${ }_{215}$ spiral. Results from other MHD models show qualitatively similar magnetopause shapes.

We display results from three numerical (SWMF, LFM, and SPBU15 with $\Delta B=0$ ) and two enical (S98, W13) models in the equatorial and noon-meridional planes in ${ }_{218}$ Figure 2. In the subsolar region, the result from the S98 model nearly coincides with the predictus of the SWMF and SPBU15. The LFM model predicts the magnetopause 
${ }_{221} \simeq 1.5 R_{E}$ larger than in the other models. The S98 model is axisymmetric, therefore it

${ }_{222}$ does not reproduce the cusp indentations, while the other models do predict this feature,

${ }_{223}$ although the size and depth of the indentations differ between each other. In the low-

${ }_{224}$ latitude region, the SWMF and SPBU15 predict that distances to the magnetopause are

${ }_{225}$ slightly on the dusk than on the dawn side (compare to the axisymmetric S98

226 model). She nly possible reason for this difference in the MHD simulations with no

${ }_{227}$ dipole tilt and a uniform ionospheric conductance is the Parker spiral IMF orientation.

${ }_{228}$ In this case increase of the magnetic field near the magnetopause is slightly larger

${ }_{229}$ downstream of the quasi-perpendicular bow shock (on the dusk flank) resulting in the ()

${ }_{230}$ asymmetric magnetopause compression. The LFM model does not predict this feature

${ }_{231}$ because it fras been run with the solar wind condition $B_{x}=0$ which is the default option ${ }_{232}$ used in CMCsimulations.

${ }_{233}$ In gene al $\mathrm{t}$ e differences between the models in Figure 2 do not exceed $1 \mathrm{R}_{E}$, except ${ }_{234}$ for the neyuts of the W13 model near the $z=0$ plane and of the S98 model near and ${ }_{235}$ behind 0 . In that region the difference amounts to $\simeq 1.5 R_{E}$.

\subsection{Maghotopause reference points}

We are going to quantify the model predictions using radial distances to the magne${ }_{237}$ topause a several selected points. We find the magnetopause intersections with the $\mathrm{x}, \mathrm{y}$, and $\mathrm{z}$ axedrat is, the subsolar point and four points in the terminator plane. We do not ${ }_{239}$ address the tai ward locations, because the nightside magnetopause is poorly determined ${ }_{240}$ in MHD sin rations and the empirical models are based on much less observations in that ${ }_{241}$ region. 
Table 2 shows the magnetopause positions (in $R_{E}$ ) at the reference points in the $B z 0$ case as predicted by the empirical models. $R_{x}$ corresponds to the subsolar point, $R_{y}$ and $R_{-y}$ correspond to the y axis crossings on the dusk and dawn flanks respectively. As mentioned above, the MHD models predict $\left|R_{y}\right|<\left|R_{-y}\right|$ because the IMF is directed along ther spiral. From the empirical models, only the L10 model is asymmetric with resp theth the $y=0$ and the $z=0$ planes and predicts a similar difference $\left(R_{y}+R_{-y}=-0.5 R_{E}\right)$. The L10 model also predicts that $R_{z}$ is significantly smaller than both $R_{y}$ a $\left|R_{y}\right|$, which is the effect of the cusp indentations. The differences between $R_{z}$ and $R_{\text {in }}$ the $\mathrm{L} 10$ model is small, about $0.1 \mathrm{R}_{E}$, therefore we do not discuss it.

Results of MHD models in the $B z 0$ run are collected in Table 3 . The difference in $R_{x}$ between the SWM and LFM/OpenGGCM is $0.7 R_{E}$, i.e. several times larger than the SWMF grasolution of $\simeq 0.125 R_{E}$. The SWMF, SWMF-CRCM, OpenGGCM, and SPBU15 r rijt a moderate dawn/dusk asymmetry in the flank locations (mentioned above), a negative $\left(R_{y}+R_{-y}\right)$ ranging from -0.8 to $-0.3 R_{E}$.

We cartify the effect of east-west elongation (or equivalently north-south contraction) in the terminator plane related to the magnetopause indentations near the cusps using the aneter $r_{y z}=\left(R_{y}-R_{-y}\right) /\left(R_{z}-R_{-z}\right) \cdot r_{y z}>1$ for the asymmetric empirical and all MHD models, except the OpenGGCM. We get $r_{y z}=1.12$ and 1.19 for the empirical L10 and 13 models, $r_{y z}=1.11,1.13$ and 1.10 for the SWMF, SWMF-CRCM and $+$

LFM modespectively, and $r_{y z}=1.05$ for the SPBU15 (without taking into account the $\mathrm{RC})$.

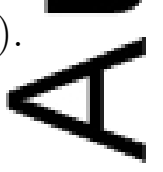




\subsection{Verification of model predictions for several selected events}


${ }_{285}$ we assume that $R_{x} \sim P_{d y n}^{-1 / 6}$ and the magnetopause shape in the subsolar region coincides

${ }_{286}$ with the $\mathrm{S} 98$ model. Thus we take into account variations of the radial distance with $P_{d y n}$

${ }_{287}$ and solar zenith angle, but not with $B_{z}$. The IMF $B_{z}$ varies between -1.1 and $5.5 \mathrm{nT}$, and

${ }_{288}$ the average $B_{z}$ equals $2.1 \mathrm{nT}$ for all events.

${ }_{289}$ We get of estimated $R_{x}^{\text {cor }}$ ranging from 10.57 to $11.88 R_{E}$ with an average ${ }_{290}<R_{x}^{c o r}>11 \pm 0.3$. Apparently we cannot completely rule out the effect of the dipole ${ }_{291}$ tilt which may significantly (at $\simeq 1 R_{E}$ for $\Psi=10^{\circ}$ ) change $R_{x}$ according to Wang ${ }_{292}$ et al. [2012]. 5 the event 11.10.2009, we have the smallest magnitude of the tilt angle ${ }_{293} \Psi=-1.0^{\circ}$ and $B_{z}$ close to zero $\left(B_{z}=-0.8 \mathrm{nT}\right)$, and we obtain the largest $R_{x}^{\text {cor }}=11.68$ ${ }_{294} R_{E}$ (average between two values). On the contrary, the smallest $R_{x}^{c o r}=10.57 R_{E}$ is 295 obtained in 08.02.2013, when the tilt angle magnitude is largest $\left(\Psi=-7.0^{\circ}\right)$ even for ${ }_{296}$ positive $\left.B_{(R}=4.8 \mathrm{nT}\right)$.

${ }_{297}$ In the thing above, we use the solar wind dynamic pressure calculated from the ${ }_{298}$ proton as given by OMNIWeb. We assume that the input parameter $P_{d y n}$ in 299 most en all MHD models corresponds to the proton pressure. If we take into 300 account that about $4 \%$ of solar particles are the $\mathrm{He}^{+2}$ ions, the dynamic pressure should 301 be multiplifany 1.16 that results in a larger $R_{x}^{c o r}$. In the last case, $\left\langle R_{x}^{c o r}>=11.5 \pm 0.3\right.$. 302 Plots of $R^{\text {cor }}(\Psi), R_{x}^{c o r}\left(B_{z}\right)$, and $R_{x}^{c o r}(D s t)$ (not shown) reveal that $R_{x}^{\text {cor }}$ and $\Psi$ are зоз anticorrelated for these events, but the dependencies $R_{x}^{c o r}\left(B_{z}\right)$ and $R_{x}^{c o r}(D s t)$ are not 304 clearly dened due to poor statistics. We discuss these results below.

\subsection{Diffences between northward and southward IMF cases}

It is known that the subsolar magnetopause moves earthward when the IMF rotates

from northward to southward. This effect can be explained either in terms of the mag- 
344 a small

Now let us consider the magnetopause shape in the terminator plane. It is known that the magnetopause flaring angle increases for a southward IMF, however this effect is rather weak in the axisymmetric S98 model. In fact this effect is strongly non-axisymmetric: the MHD simulations presented below show that the distance to the magnetopause increases along ther than along the $y$ axis when IMF $B_{z}$ turns southward. Note that the position o $R_{z}$ the magnetopause intersections with the $z$ axis) should always lie tailward of the cusp, as predicted by most models. For northward IMF conditions, magnetic reconnection ocgur at the high-latitude magnetopause where the boundary moves earthward. For southyard IMF conditions, magnetic field lines reconnected at the dayside magneC) topause convect tailward and accumulate the magnetic flux in the tail lobes [Dungey, $\longrightarrow$ 1961]. Consequently, the magnetopause radius tailward of the cusps should increase for southwardIME in agreement with previous studies [Boardsen et al., 2000].

Only tw 1 irical models, W13 and SG15, are really able to reproduce this effect predicting -1.16 and $-0.50 R_{E}$ respectively. On the contrary, the L10 model predicts

352 $0.2 R_{E}$ for the assumed solar wind conditions. 


\subsection{Effect oI the dipole tilt}


${ }_{380}$ for $\Psi=$ was predicted by Sotirelis and Meng [1999] (see Figure 9 in their paper),

381 although the effect becomes more significant $\left(\simeq 1 R_{E}\right)$ for $\Psi=-35^{\circ}$.

382

383

The dipole tilt effect can also be estimated from models which calculate the magnetopause position using the pressure balance condition. In particular, Olson [1969] found that the subsolar distance decreases with increasing tilt angle, but this effect is relatively weak. The increase of $\Psi$ from $0^{\circ}$ to its maximum of $35^{\circ}$ results in $\Delta R_{x} \leq 0.03 R_{x}$, i.e. for $R_{x}=11$ gives $\Delta R_{x} \simeq-0.3 R_{E}$. Similarly, a small tilt effect at the subsolar point s enter

Thus theot models predict a weaker dipole tilt effect in the subsolar region than that predicted by the W13 model. However, only three empirical models (B00, L10 and W13) (

in principle are able to estimate this effect at the subsolar point. From these models, 35 B00 was espectally developed for this region and therefore may be more accurate, and its result is in armediate between two others.

Near an 1 ind the cusps, the tilt effect predicted by both the L10 and W13 models is enhance the nose B00 model does not work at high latitudes above $80^{\circ}$ ). Behind the cus sign, i.e. it is negative below and positive above the equatorial plane. This qualitatively agrees with the previous simulations [Sotirelis and Meng, 1999].

\subsection{Effet o the ring current}

As desc (bed in Section 2.3, we can add the RC magnetic field to the dipole field in the region outide RC. As expected, the magnetopause distance increases in all directions $(x, y, z)$ in the uns with the $\mathrm{RC}$, because the addition of the $\mathrm{RC}$ is effectively equivalent to an increase the geodipole moment and, hence, increases the magnetic field on the inner side of the magnetopause. Table 3 contains the corresponding values at the reference 
${ }_{396}$ points in the runs of the SPBU15 with the $\mathrm{RC}$ corresponding to $\Delta B=-20 \mathrm{nT}$ (run

${ }_{415} \mathrm{nT}$. 
${ }_{419}$ enhancement of the magnetospheric magnetic field is even a little larger than that for the ${ }_{420} \mathrm{RC}$ with $\Delta B=-60 \mathrm{nT}$ in our case $\left(R_{x}=11.5 R_{E}\right)$. This difference is explained by ${ }_{421}$ different assumptions about the location of the RC.

${ }_{422}$ Our numerical estimations agree with observations in Hayosh et al. [2005]. Hayosh et al. ${ }_{423}$ [2005] the difference between the model and observed magnetopause positions ${ }_{424}$ with the st dex and found that the magnetopause moves outward on average by 0.5 ${ }_{425} \quad R_{E}$ as $D s$ changes from +20 to $-60 \mathrm{nT}$. This dependence of $R_{x}$ on $D s t$ is only slightly ${ }_{426}$ weaker that obtained in our work. However, it should be taken into account that ${ }_{427}$ Hayosh et al [2005] analyzed the tail region between $\mathrm{X}=-19$ and $\mathrm{X}=0 R_{E}$. Note also ${ }^{428}$ that the observed ground disturbance $(D s t)$ is, roughly, a factor of 1.3 larger than the ${ }_{429} \mathrm{RC}$ magnetreffect $\Delta B$ used in our study, which is quantified in the equation for the ${ }_{430} "$ corrected ${ }_{\Delta s} t^{*}=0.8 D s t-13 \sqrt{P_{d y n}}$ [e.g., Tsyganenko and Sitnov, 2005]. Therefore ${ }^{431}$ taking int $\mathrm{Cc}$ unt the telluric currents, the correspondence between results of Hayosh et ${ }_{432}$ al. (200, and ours becomes even better.

Both $R_{z}$ in the MHD simulations also increase with the RC, but $R_{y}$ grows faster ${ }_{434}$ than $R_{x}$ and $R_{z}$. As a result, the east-west elongation parameter $r_{y z}$ increases from 1.05 ${ }^{435}$ for $\Delta B=1.07$ for $\Delta B=-60 \mathrm{nT}$.

The effect of the RC should be reproduced in SWMF-CRCM simulations. Indeed the ${ }_{437}$ SWMF-CRCM predicts a more distant magnetopause than the SWMF as shown in the ${ }_{438}$ first two of Table 3 . In particular, $R_{x}$ is larger by $0.2 R_{E}, R_{y}\left(R_{-y}\right)$ by 0.7 ${ }_{439}(0.6) R_{E}$, and $R_{z}$ by $0.3 R_{E}$. Thus the CRCM makes similar or larger changes in the 440 magnetoparance than the $\mathrm{RC}$ with $\Delta B=-20 \mathrm{nT}$ in the SPBU-RC20 run, but ${ }_{441}$ always smaller changes than in the SPBU-RC60 run (the last predicts a difference of 
${ }_{442} 0.6 R_{E}$ in $R_{x}$ and $0.9 R_{E}$ in $R_{y}$ as mentioned above). The calculated Dst index in the

${ }_{443} \mathrm{SWMF-CRCM}$ run is $4 \mathrm{nT}$.

\section{Discussion and conclusions}

The magnetopause positions can be predicted using both empirical and analytical mag-<smiles>[13CH][13CH3]</smilesnetopause 1 els and global MHD models. This paper compares results from different (110y) models for the stationary typical solar wind conditions under which both empirical and

${ }_{447}$ MHD mocin should work rather well. We search for systematic differences between ax${ }_{448}$ isymmetricon non-axisymmetric empirical and MHD models and suggest explanations ${ }_{449}$ for these 4 ences. Additionally, we find several subsolar magnetopause crossings to ${ }_{450}$ compare with he model predictions.

${ }_{451}$ We sup ose that both empirical and MHD models may have disadvantages in predicting ${ }_{452}$ the three-dimasional magnetopause. Empirical models make a priori assumptions about ${ }_{453}$ the magnetopause shape: some of them relate the radial distance to the solar zenith ${ }_{454}$ angle usino functional forms (e.g., the S98 and L10 models), while others set several ${ }_{455}$ fitting parameters based on implicit assumptions about most probable (rather smooth) 456 magnetopause shape (W13). Most empirical models, except the recent L10 and W13, are ${ }_{457}$ axisymme nd, hence, are inaccurate near the terminator plane. The axisymmetric ${ }_{458}$ models do not reproduce the cusp indentations, but also may underestimate the radial ${ }_{459}$ distance 1 ear the equatorial plane because of the averaging. Empirical models, again ${ }_{460}$ except L11 461 Wang 2013] found that a tilt angle increase from $0^{\circ}$ to $10^{\circ}$ under low solar wind ${ }_{462}$ dynamic pressure results in a shift of the subsolar point by $\sim 1 R_{E}$ earthward and causes ${ }_{463}$ a significant deformation of the dayside magnetopause in the $x z$ plane. In this paper, we 
${ }_{464}$ compare the magnetopause positions in the meridional plane for tilts $15^{\circ}$ and $0^{\circ}$ predicted

${ }_{465}$ by the nose B00, L10, W13, and two MHD models and find that the all models except

${ }_{466}$ W13 predict a relatively small difference $\Delta R$ between $\Psi=15^{\circ}$ and $0^{\circ}$ in the subsolar

${ }_{467}$ region, although $\Delta R$ increases near the cusps. We cannot decide which predictions are

${ }_{468}$ more acthout additional model validation in the future.

MHD nodel do not include kinetic effects, but we can specify which kinetic factors

${ }_{470}$ are important for correct magnetopause predictions. The magnetopause position depends

${ }_{471}$ on the RCwich is not properly described by the MHD codes. We estimate the effect

${ }_{472}$ of the RCat the subsolar magnetopause by modifying the SPBU15 code and making $\overbrace{}^{a t}$

${ }_{473}$ simple calculations, based on assumption of a purely dipole internal field. We find that an

${ }_{474}$ assumed symmetrical RC with $\Delta B=-20 \mathrm{nT}$ at the Earth and $R_{\mathrm{RC}}=5.5 R_{E}$ enhances

475 the subsol $\mathrm{r}$ distance by $\simeq 0.23 R_{E}$, while a stronger current with $\Delta B=-60 \mathrm{nT}$ enhances ${ }_{476} R_{x}$ by $\simeq 6 \mathbb{R}$. Since a strong $\mathrm{RC}(\Delta B<-60 \mathrm{nT})$ occurs only during magnetic storms,

${ }_{477}$ the corran of the subsolar distance on the RC effect in MHD results usually should not 48 exceed $($ However, this estimate depends on the radius of the RC. A symmetrical RC ${ }_{479}$ located farther from the Earth results in a stronger effect at the subsolar magnetopause.

${ }_{480}$ Moreover, shape of the ring current in the dayside magnetosphere is still not well ${ }_{481}$ established and may differ from a torus [Kirpichev and Antonova, 2014; Andreeva and Tsyganenko, 2016] which would also influence the magnetopause position.

Global models coupled with the inner magnetospheric models, e.g., with the ${ }_{484}$ RCM or CRCM, may better reproduce the location of the magnetopause. In particular, ${ }_{485}$ the results the SWMF-CRCM in the $B z 0$ case approach the results of the empirical L10 model closer than the results of SWMF without the ring current model. However, 
${ }_{510}$ the plasma parameters from the solar wind monitors near the L1 point and close to the ${ }_{511}$ bow shock (e.g., from ACE and THEMIS) are often intercalibrated, which eliminates ${ }_{512}$ differences between them.

${ }_{513}$ Samsonov et al. [2012] showed that the total pressure varies along the Sun-Earth line ${ }_{514}$ across thetosheath and these variations depend on the IMF orientation. Shue and ${ }_{515}$ Chao 201 ex ressed the magnetopause pressure balance in the form $\left(f B_{e} / R_{x}^{3}\right)^{2} \sim k P_{d y n}$, ${ }_{516}$ where $B_{e}$ the magnetic field strength on the equatorial surface of the Earth, $f$ is the 517 coefficient cefting the role of magnetopause currents, and the coefficient $k$ denotes the ${ }_{518}$ fraction of the solar wind dynamic pressure applied to the magnetopause. Shue and ${ }_{519}$ Chao $[2013$ showed that $f$ can vary from $\sim 2.07$ to 2.55 , and $k$ can vary from 0.74 to 0.94 , ${ }_{520}$ depending orr the IMF $B_{z}$ and solar wind dynamic pressure. MHD models self-consistently ${ }_{521}$ take into bount both the changes of the total pressure across the magnetosheath and ${ }_{522}$ the magnfobalse deformation (since $f$ varies depending on the magnetopause shape and ${ }_{523}$ electric Empirical models are based on measured upstream parameters and ${ }_{524}$ observe lopause locations, consequently, both $f$ and $k$ variations are included but ${ }_{525}$ they cannet be separated.

${ }_{526}$ MHD m predict the thermal pressure in the dayside outer magnetosphere $p \simeq 0.1$ ${ }_{527} \mathrm{nPa}$ which is in general agreement with quiet-time observations [e.g., Phan et al., 1994; Shue and Chao, 2013]. Simulations using an anisotropic MHD model (anisotropic MHD '十10 equations local magnetosheath model presented by Samsonov et al. [2007]) (not ${ }_{530}$ shown) indicat that anisotropic pressures only slightly change the subsolar magnetopause ${ }_{531}$ distance. 1 agrees with global anisotropic MHD results of the uncoupled BATS-R-US ${ }_{532}$ (later developed to SWMF) code [Meng et al., 2013], while the subsolar point predicted 
${ }_{533}$ by the anisotropic BATS-R-US coupled with both RCM or CRCM is $\sim 0.4-0.5 R_{E}$ closer ${ }_{534}$ to the Earth than that predicted by the corresponding isotropic code.

Comparing predictions of empirical and MHD models, we emphasize several items.

\section{Positions of the subsolar point in the $B z 0$ case.}

The distance to the subsolar point from all axisymmetric empirical models (PR96, K 98, 98, S98) is $11.1 R_{E}$, which agrees with both the average subsolar position obtained for seven selected events $\left(11.2 R_{E}\right)$ and $R_{x}$ predicted by the SWMF and the 3

SPBU-R2Qco (with the added symmetrical $\mathrm{RC}$ with $\Delta B=-20 \mathrm{nT}$ and $R_{\mathrm{RC}}=5.5 R_{E}$ ). Other MHD cedes, LFM and OpenGGCM, predict a smaller distance $R_{x}=10.4 R_{E}$. The क

difference between MHD predictions may be explained by different boundary conditions $\longrightarrow$

at the low-artude boundary, affecting the plasma pressure inside the magnetopause.

Colobal non-axisymmetric empirical models (L10 and W13) predict $R_{x}=11.47$, and 12.60 r spectively, i.e., larger than both axisymmetric empirical and MHD models.

To check prediction, we have additionally calculated $R_{x}$ using the local (for the nose region) model of Boardsen et al. [2000] and obtained $11.84 R_{E}$, i.e. between the L10 and W13 results. As discussed above, the axisymmetric empirical models (e.g., PR96 or S98) do 100 ake into account the dipole tilt effect and therefore may underestimate the subsolar distance for zero tilt. In the selected events, the average tilt angle is $|\Psi|=5.3^{\circ}$, ${ }_{51}$ i.e., the average $R_{x}$ may still differ from that in the untilted case $\Psi=0^{\circ}$. In event with $+$

52 $\Psi$ closest ${ }_{553}$ the subsolar distance for several reasons, such as the RC effect or depressed solar wind ${ }^{54}$ dynamic prosure upstream of the bow shock. 
0.93 in the SWMF and LFM models respectively. The L10 model predicts insignificant changes in $r_{y z}$ for the $B z+$ and $B z$ - cases. Thus only one empirical model (W13) may in principle correctly predict the dawn-dusk elongation and its variations with the $B_{z}$ sign, and the MHD model predictions differ from each other.

Compdictions of MHD models with the ring current (SWMF-CRCM, SPBURC20, an SPBU-RC60) and non-axisymmetic empirical models (L10, W13, and SG15 reference points in the terminator plane, we get a relatively good agreement between th In particular, $R_{z}$ in the case $B z 0$ is between 14.6 and $15.6 R_{E}$ as confirmed by all these models. The range of $R_{y}$ predicted by SWMF-CRCM, SPBU-RC and L10 is from 15.9 to $16.4 R_{E}$, while W13 yields $17.9 R_{E}$. The magnitude $R_{-y}$ is about $0.5 R_{E}$ larger thanrs.

\section{Compatison between northward and southward IMF cases.}

The diff $r$ netween the $B z+$ and $B z-$ cases is evaluated by means of the parameter $\left.\Delta R_{x}=\sqrt{z+}\right)-R_{x}(B z-)$. Its value varies from $0.28 R_{E}$ in the $\mathrm{S} 98$ model to 0.89 and 0.95 in 13 and P98 models. The MHD models predict $\Delta R_{x}$ within a narrower (or the same) range of values, e.g., $0.1 R_{E}$ in SWMF and $0.6 R_{E}$ in LFM. In the MHD codes, ange

the $\Delta R_{x}$ poty bly depends on the magnitude of magnetospheric-ionospheric currents.

As mentioned above, $R_{z}$ decreases from southward to northward IMF, however only the W13 and SGI5 empirical models predict such a decrease, with $\Delta R_{z}=-1.16$ and -0.50 $+$

$R_{E}$ respectured All MD models predict negative $\Delta R_{z}$, e.g., $-1.3 R_{E}$ in SWMF and -2.5 $R_{E}$ in LFM, and $\left|\Delta R_{z}\right|$ is larger than in the empirical models. The $\Delta R_{y}$ is relatively small both in empsical and in MHD models. 
A slightly larger compression on the dusk flank due to the Parker spiral IMF.

When the IMF is oriented along the Parker spiral, the dusk magnetosphere lies downstream of the quasi-perpendicular bow shock, and the dawn magnetosphere lies downstream since the magnetosheath magnetic field is larger downstrea from the quasi-perpendicular bow shock, the total pressure on the duskside magnetopause is higher than that on the dawnside magnetopause. Consequently the magnetopas distance is smaller on the dusk side than on the dawn side. Among the empirical models, only L10 is able to reproduce this effect. W13 model uses only the क

dynamic pressure and $B_{z}$ in the solar wind data and therefore assumes symmetry across $\longrightarrow$

the noon-mrerrdional plane. On the contrary, all MHD models, except LFM, predict this difference LFM model does not predict this effect because of the fixed solar wind condition $B_{x}=0 \mathrm{~s} d$ in the runs presented here. The L10 model predicts $R_{y}+R_{-y}=-0.5$ $R_{E}$, very smiar to the predictions of the SWMF and SPBU15 codes.

\section{Diffe metween the empirical and MHD models.}

Axisympetric empirical magnetopause models do not reproduce the three-dimensional magnetopand lose information due to the tilt angle averaging. The position of the subsolar pointin the axisymmetric models (PR96, S98) is closer to the Earth than in the non-axisymmetric models (B00, L10, W13) for $\Psi=0^{\circ}$. In general, all the reference points $\left(R_{x}, R_{y}, R_{-1}\right.$ dicted by the non-axisymmetric models are also farther from the Earth than the corresponding points predicted by the numerical models (SWMF, LFM) in the $B z 0$ and 1 cases, i.e., the MHD codes most likely underestimate the magnetopause distance. However, predictions of the SPBU15 code with the relatively strong RC with 
${ }_{623} \Delta B=-60 \mathrm{nT}(\mathrm{SPBU}-\mathrm{RC} 60)$ are close to the L10 results in $R_{x}, R_{y}$ and $R_{-y}$ in the $B z 0$

${ }_{624}$ case, while $R_{z}$ in the MHD results on $0.5 R_{E}$ larger than in L10, but nearly equal to the

${ }_{625}$ prediction of the SG15 empirical model developed for the high-latitude magnetopause.

${ }_{626}$ The magnetopause position predicted by the SWMF coupled with the CRCM is closer to

${ }_{627}$ the L10 Lun that in the uncoupled SWMF, but the magnetopause distance in the

${ }_{628}$ SWMF-C CMun is still slightly underestimated in comparison with L10.

${ }_{629}$ Summaning the large amount of information in this paper, we still cannot give a

${ }_{630}$ positive and to the question in the title. Comparing MHD models in which the ring

${ }_{631}$ current magnetic field is taken into account (BATSRUS-CRCM, SPBU-RC) with the क

32 empirical non-axisymmetric L10 model, we find that the differences in the reference point $\longrightarrow$

positions preurcted by these models are relatively small. Therefore we assume that these

prediction indicate the actual magnetopause position in the Bz0 case. However, the large difference 20 en L10 and W13 results $\left(>1 R_{E}\right)$ near the equatorial plane requires further investiganen some respects, the W13 model makes more reasonable predictions, e.g. when it fully reproduces the effect of a southward IMF at the terminator plane.

It is also important to note that W13 employs the largest database, including crossings

from both rognt and old missions, because some missions (THEMIS, MMS) have an apogee in the subsolar region near $12 R_{E}$ and may miss more distant magnetopause crossings. We Delieve that the role of the dipole tilt on the magnetopause position is still

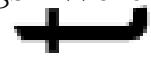

not compunderstood. Furthermore, the next generation of magnetopause models should treat magnetopause crossings for nearly radial IMF separately, because these are times when magnetosheath pressure becomes significantly lower than the solar wind dynamic pressure [Suvorova and Dmitriev, 2015]. If the number of such events in a 
${ }_{646}$ magnetopause crossings database is relatively large, the models which do not consider

the IMF cone angle as an input parameter will overestimate the magnetopause distance.

Finally, we hope that the results of our work can help to develop a new three-dimensional empirical magnetopause model which can give a positive answer to the question in the title.

Acknovadoments. This work was supported by the Russian Foundation for Basic Research rant 14-05-00399. The work by E.G. was supported by RFBR grant 14-0531380. J. Safínková and Z. Němeček thank to the Czech Grant Agency for support under Con 14-19376S. AAS thanks Prof. Victor Sergeev for valuable comments. We thank Dr.y li Wang for providing results of the W13 model. Simulation results were provided by the Community Coordinated Modeling Center (http://ccmc.gsfc.nasa.gov) at Goddard Space Flight Center. THEMIS data are available from the Coordinated Data Analysis 20 CDAWeb) and THEMIS web site (http://themis.igpp.ucla.edu). OMNI data are avaigable from OMNIWeb service (http://omniweb.gsfc.nasa.gov).

\section{Referencis}

${ }_{60}$ Andreeva. W. and N. A. Tsyganenko (2016), Reconstructing the magnetosphere from data usi radial basis functions, J. Geophys. Res. Space Physics, 121, 2249-2263, doi: 10.1002 2015JA022242.

Beard, D. B. 960), The interaction of the terrestrial magnetic field with the solar corpuscular tration, J. Geophys. Res., 65(11), 3559-3568, doi:10.1029/JZ065i011p03559.

Boardsen, S. A., T. E. Eastman, T. Sotirelis, and J. L. Green (2000), An empirical model of the high-latitude magnetopause, J. Geophys. Res., 105(A10), 23,193-23,219, 
doi:10.1029/1998JA000143.

Brackbill J. U. and D. C. Barnes (1980), The effect of nonzero $\nabla \cdot \mathbf{B}$ on the numerical solution of the magnetohydrodynamic equations, J. Comput. Phys. 35, 426-430, doi: 10.1016/0021-9991(80)90079-0.

Chapma hi V. C. Ferraro (1931), A new theory of magnetic storms, Terr. Magn. Atmos. Elect.. 36(2), 77-97, doi:10.1029/TE036i002p00077.

De Zeeuw D., S. Sazykin, R. Wolf, T. Gombosi, A. Ridley, and G. Toth (2004), Coupling of a global $\mathrm{HD}$ code and an inner magnetosphere model: Initial results, J. Geophys. Res., 109(A12), A12219, doi:10.1029/2003JA010366.

Dmitriev, A. V., and A. V. Suvorova (2000), Three-dimensional artificial neural network more of the dayside magnetopause, J. Geophys. Res., 105, 18,909-18,918, doi: 10.1029 2000JA900008.

Dungey IO(1961), Interplanetary magnetic field and the auroral zones, Phys. Rev. Lett.

Elsen,

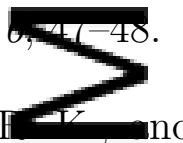

n. M. Winglee (1997), The average shape of the Magnetopause: A comparison of three-dimensional global MHD and empirical models, J. Geophys. Res., 102(A3) 9-4819, doi:10.1029/96JA03518.

Fairfield, D_H. (1971), Average and unusual locations of the Earth's magnetopause and bow snock, Geophys. Res., 76(28), 6700-6716, doi:10.1029/JA076i028p06700.

Fairfield, Baumjohann, W., Paschmann, G., Lühr, H., Sibeck, D. G. (1990), Upstream pressure variations associated with the bow shock and their effects on the magnetosphen L. Geophys. Res., 95(A4), 3773-3786, doi:10.1029/JA095iA04p03773.

Fok, M., R. A. Wolf, R. W. Spiro, and T. E. Moore (2001), Comprehensive com- 
putational model of Earth's ring current, J. Geophys. Res., 106, 8417-8424, doi: 10.1029/2000JA000235.

García, K. S., and W. J. Hughes (2007), Finding the Lyon-Fedder-Mobarry magnetopause: A statistical perspective, J. Geophys. Res., 112, A06229, doi:10.1029/2006JA012039.

Glocer, Fok, X. Meng, G. Tóth, N. Buzulukova, S. Chen, and K. Lin (2013), CRCM PATS-R-US two-way coupling, J. Geophys. Res., 118, 1635-1650, doi: 10.1002 jgra.50221.

Gombosi, J. G. Tóth, D. L. De Zeeuw, K. C. Hansen, K. Kabin, K. G. Powell (2002), Semirelativistic magnetohydrodynamics and physics-based convergence acceleration, $J$. Comput. Phys., 177, 176-205, doi:10.1006/jcph.2002.7009.

Gordeev, Sergeev, I. Honkonen, M. Kuznetsova, L. Rastätter, M. Palmroth, P. Janhun_G Tóth, J. Lyon, and M. Wiltberger (2015), Assessing the performance of commur it -available global MHD models using key system parameters and empirical relationdens, Space Weather, 13, doi:10.1002/2015SW001307.

Hayosh Němeček, J. Šafránková, and G. N. Zastenker (2005), Variations of the magnetgsheath ion flux and geomagnetic activity, Adv. Space Res., 36(12), 2417-2422, doi:10.10 asr.2003.08.082.

Hellinger, P_P. Travnicek, J. C. Kasper, and A. J. Lazarus (2006), Solar wind proton temperature anisotropy: Linear theory and WIND/SWE observations, Geophys. Res. $1+3$

Lett., 30ـ101, doi:10.1029/2006GL025925.

Hill, T. W., and M. E. Rassbach (1975), Interplanetary magnetic field direction and the conrs kation of the day side magnetosphere, J. Geophys. Res., 80(1), 1-6, doi: 10.1029/JA080i001p00001. 
J. Geophys. Res., 69(7), 1169-1179, doi:10.1029/JZ069i007p01169.

Meng, X., G. Tóth, A. Glocer, M.-C. Fok, and T. I. Gombosi (2013), Pressure anisotropy in global magnetospheric simulations: Coupling with ring current models, J. Geophys. Res., 118, 5639-5658, doi:10.1002/jgra.50539.

Merkin, and J. G. Lyon (2010), Effects of the low-latitude inospheric boundary condition o the global magnetosphere, J. Geophys. Res., 115, doi:10.1029/2010JA015461.

Merkin, G., Lyon, J. G., and Claudepierre, S. G. (2013), Kelvin-Helmholtz instability of the magneric boundary in a three-dimensional global MHD simulation during northward IMF conditions, J. Geophys. Res., 118, 5478-5496. doi:10.1002/jgra.50520. C)

Němeček, Z., J. Šafránková, O. Kruparova, L. Přech, K. Jelínek, Š. Dušík, J. Šimunek, K. Grygorov, and J.-H. Shue (2015), Analysis of temperature versus density plots and their relion to the LLBL formation under southward and northward IMF orientations, J. Geop?yes. Space Physics, 120, 3475-3488, doi:10.1002/2014JA020308.

Olson, (1969), The shape of the tilted magnetopause, J. Geophys. Res., 74(24), 5642-10.1029/JA074i024p05642.

Palmroth, M., T. I. Pulkkinen, P. Janhunen, and C.-C. Wu (2003), Stormtime energy trang in global MHD simulation, J. Geophys. Res., 108(A1), 1048, doi: 10.1029/2002JA009446.

Pembroke, A., F. Toffoletto, S. Sazykin, M. Wiltberger, J. Lyon, V. Merkin, and P. Schmitt 2), Initial results from a dynamic coupled magnetosphere-ionosphere-ring current model, J. Geophys. Res., 117, A02211, doi:10.1029/2011JA016979.

Petrinec, P. P. Song, and C. T. Russell (1991), Solar cycle variations in the size and shape of the magnetopause, J. Geophys. Res., 96(A5), 7893-7896, doi: 
magnetosphere, Planet. Space Sci., 14, 223-253, doi:10.1016/0032-0633(66)90124-3.

Suvorova, A. V., and A. V. Dmitriev (2015), Magnetopause inflation under radial IMF: Comparison of models, Earth and Space Science, 2, 107-114, doi: 10.1002/2014EA000084.

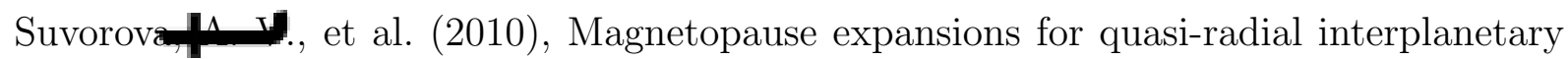
magnet fied: THEMIS and Geotail observations, J. Geophys. Res., 115, A10,216, doi:10.1029/2010JA015404.

Tanaka T. (1994), Finite volume TVD scheme on an unstructured grid system for threedimensional_MHD simulations of inhomogeneous systems including strong background c

potential field, J. Comp. Phys., 111, 381-389, doi:10.1006/jcph.1994.1071.

Toffoletto, T.R., S. Sazykin, R. W. Spiro and R. A. Wolf (2003), Modeling the Inner Magnet anhere using the Rice Convection Model (review), Space Sci. Rev., 108, 175196. 0

Tóth, G. al. (2005), Space Weather Modeling Framework: A new tool for the space scien unity, J. Geophys. Res., 110, A12226, doi:10.1029/2005JA011126.

Tóth, G. et al. (2012), Adaptive Numerical Algorithms in Space Weather Modeling, Journal jomputational Physics, 231, 870, doi:10.1016/j.jcp.2011.02.006.

Tsyganenko_N A., and D. G. Sibeck (1994), Concerning flux erosion from the dayside magnetosphere, J. Geophys. Res., 99(A7), 13425-13436, doi:10.1029/94JA00719.

Tsyganenl. A. (1995), Modeling the Earth's magnetospheric magnetic field confined within realistic magnetopause, textitJ. Geophys. Res., 100(A4), 5599-5612, doi: $10.1029 / 9$ LA 03193.

${ }_{227}$ Tsyganenko, N. A. (1996), Effects of the solar wind conditions on the global magne- 
tospheric configuration as deduced from data-based field models, in European Space Agency Publication, ESA SP, 389, 181-185.

Tsyganenko, N. A., and M. I. Sitnov (2005), Modeling the dynamics of the inner magnetosphere during strong geomagnetic storms, J. Geophys. Res., 110, doi: 10.10201/JA010798.

Tsyganen D. A., and V. A. Andreeva (2015), A forecasting model of the magnetosphere griven by an optimal solar wind coupling function, J. Geophys. Res., 120, doi: $10.1002 / 2015 \mathrm{JA} 021641$.

Wang, Y. D G. Sibeck, J. Merka, S. A. Boardsen, H. Karimabadi, T. B. Sipes, J. め Šafránková,KK. Jelínek, and R. Lin (2013), A new three-dimensional magnetopause model wrup support vector regression machine and a large database of multiple spacecraft ob Corvations, J. Geophys. Res., 118, 2173-2184, doi:10.1002/jgra.50226.

Wolf, R. .R W. Spiro, and F. J. Rich (1991), Extension of the Rice Convection Model into then-latitude ionosphere, J. Atm. Terrest. Phys., 53, 817-829.

Xi, S. Iko, B. Zhang, O. J. Brambles, J. G. Lyon, V. G. Merkin, and M. Wiltberger (2015), Poynting flux-conserving low-altitude boundary conditions for global agetospheric models, J. Geophys. Res. Space Physics, 120, 384-400, doi: 10.1002/2014JA020470.

Zhigulev, . . and E. A. Romishevskii (1959), Concerning the interaction of currents flowing onducting medium with the earth's magnetic field, Soviet Phys. Doklady, 5, 1001-1004 (Eng. Trans. 1960, 4, 859-862). 
Table 1. List of empirical (analytical) magnetopause models

\begin{tabular}{|l|c|c|c|c|c|c|c|r|}
\hline Model & PR96 & KS98 & P98 & S98 & B00 & L10 & W13 & SG15 \\
\hline non-axisymmetric & $\mathrm{N}$ & $\mathrm{N}$ & 1 point & $\mathrm{N}$ & $\mathrm{Y}$ & $\mathrm{Y}$ & $\mathrm{Y}$ & 1 point \\
\hline dipole tilt & $\mathrm{N}$ & $\mathrm{N}$ & $\mathrm{N}$ & $\mathrm{N}$ & $\mathrm{Y}$ & $\mathrm{Y}$ & $\mathrm{Y}$ & $\mathrm{Y}$ \\
\hline analytical form & $\mathrm{Y}$ & $\mathrm{Y}$ & $\mathrm{Y}$ & $\mathrm{Y}$ & $\mathrm{Y}$ & $\mathrm{Y}$ & $\mathrm{N}$ & $\mathrm{Y}$ \\
\hline number of crossings & 6273 & 886 & Analit.(33) & 553 & 290 & 2708 & 15,089 & 1022 \\
\hline
\end{tabular}

a ADreviations of models: PR96 [Petrinec and Russell, 1996], KS98 [Kuznetsov and Suvorova, 1998], et al., 2010], W13 [Wang et al., 2013], SG15 [Shukhtina and Gordeev, 2015].

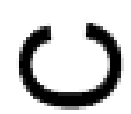

Table

Table 2. Results from the empirical (analytical) magnetopause models in the case $B z 0(N=5$ $\mathrm{cm}^{-3}, 00 \mathrm{~km} / \mathrm{s}, T=2 \times 10^{5} \mathrm{~K}, B_{y}=-B_{x}=3.5 \mathrm{nT}$ and $\left.B_{z}=0\right)$.

\begin{tabular}{|l|r|r|r|r|r|r|r|}
\hline Model & KS98 & P98 & S98 & B00 & L10 & W13 & SG15 \\
\hline$R_{x}$ & 11.45 & 10.99 & 10.90 & 11.84 & 11.47 & 12.60 & \\
\hline$R_{y}$ & & 16.33 & & 16.44 & 17.90 & \\
\hline$R_{-y}$ & & & & -16.94 & & \\
\hline$R_{z}$ & & & & 15.00 & 15.00 & 15.66 \\
\hline$R_{-z}$ & & & -14.91 & & \\
\hline
\end{tabular}

the y and $\mathrm{z}$ axes, $R_{-y}$ and $R_{-z}$ are the intersections with $-\mathrm{y}$ and $-\mathrm{z}$. All values are given in $R_{E}$.

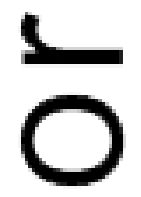

Table 3. Results from the MHD models in the run $B z 0$.

\begin{tabular}{|c|c|c|c|c|c|c|c|}
\hline Modt & VMF & SWMF-CRCM & LFM & GGCM & SPBU & SPBU-RC20 & SPBU-RC60 \\
\hline$R_{x}$ & 1.1 & 11.3 & 10.4 & 10.4 & 10.8 & 11.1 & 11.4 \\
\hline$R_{y}$ & 5.7 & 16.4 & 15.5 & 13.2 & 15.5 & 15.9 & 16.4 \\
\hline$R_{-y}$ & 16.1 & -16.7 & -15.5 & -14.0 & -16.0 & -16.4 & -16.9 \\
\hline$R_{z}$ & 4.3 & 14.6 & 14.1 & 16.5 & 15.0 & 15.2 & 15.5 \\
\hline
\end{tabular}

a probreviations 'SPBU-RC20' and 'SPBU-RC60' denote the results of the SPBU15 for

the ring current yielding $\Delta B=-20$ and $-60 \mathrm{nT}$ at the Earth. 
Table 4. Magnetopause crossings in the subsolar region observed by THEMIS

\begin{tabular}{|l|c|c|c|c|c|c|c|c|r|}
\hline Date & Time & SC & Robs & $x, y, z(\mathrm{GSM})$ & $R_{x}^{\text {cor }}$ & $P_{\text {dyn }}, \mathrm{nPa}$ & $B_{z}, \mathrm{nT}$ & $\Psi$ & Dst \\
\hline 30.09 .2009 & $16: 46$ & THE & $<11.02$ & $10.9,-1.6,-0.2$ & $10.80 / 11.67$ & $1.2 / 2.0$ & 5.5 & 7.0 & 2 \\
\hline 11.10 .2009 & $20: 01$ & THD & $>11.42$ & $11.4,-0.5,0.9$ & $11.88 / 11.49$ & $1.7 / 1.4$ & -0.5 & -1.0 & -5 \\
\hline 25.10 .2009 & $13: 05$ & THA & $<11.68$ & $11.5,-0.9,1.6$ & $10.96 / 11.72$ & $0.9 / 1.4$ & -1.1 & -6.0 & -17 \\
\hline 02.11 .2009 & $18: 52$ & THD & $\simeq 11.37$ & $10.4,-4.1,1.9$ & $\simeq 10.92$ & 1.2 & 1.2 & -6.7 & 1 \\
\hline 19.10 .2010 & $20: 03$ & THA & $\simeq 11.57$ & $11.0,2.4,2.5$ & $11.67 / 11.24$ & $1.5 / 1.2$ & 4.3 & -4.0 & -13 \\
\hline 03.11 .2010 & $15: 33$ & THE & $>11.38$ & $10.9,-1.4,2.9$ & $>11.27$ & 1.4 & 0.6 & -5.2 & -16 \\
\hline 08.02 .2010 & 14.52 & THD & $>10.37$ & $10.1,-2.1,1.4$ & $>10.57$ & 1.6 & 4.8 & -7.0 & -20 \\
\hline
\end{tabular}

a THA ITID, and THE denote THEMIS A, D, and E. Robs is the observed radial distance, $R_{x}^{c o r}$ is the corrected subsolar distance calculated for $P_{d y n}=1.34 \mathrm{nPa} . \Psi$ is the dipole tilt angle (in degrees, Dst index in nT.

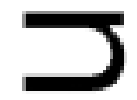

Table 5 The differences between magnetopause positions in the northward and southward cases $(R(\mathbf{X}-R(B z-))$ in the empirical models.

\begin{tabular}{|l|c|c|c|c|c|c|c|c|c|}
\hline Model & PQ96 & KS98 & P98 & S98 & B00 & L10 & W13 & SG15 & Aver. \\
\hline$\Delta R_{x}$ & 51 & 0.53 & 0.95 & 0.28 & 0.23 & 0.57 & 0.89 & & $0.57^{*}$ \\
\hline$\Delta R_{y}$ & 0.0 & 0.57 & & -0.05 & & 0.38 & -0.15 & & \\
\hline$\Delta R_{z}$ & & & & & & 0.38 & -1.16 & -0.50 & \\
\hline
\end{tabular}

* The last column contains the average $\Delta R_{x}$ for six models.

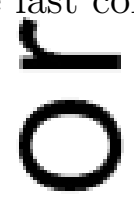

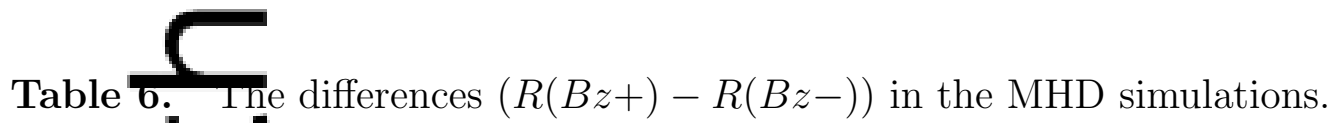

\begin{tabular}{|l|cc|c|c|r|r|}
\hline Model & & SWMF & LFM & GGCM & SPBU & Aver. \\
\hline$\Delta R_{x}$ & & 0.1 & 0.6 & 0.7 & 0.2 & 0.4 \\
\hline$\Delta R_{y}$ & 0.2 & 0.2 & 1.6 & 0.8 & $0.4^{*}$ \\
\hline$\Delta R_{z}$ & & -1.3 & -2.5 & -2.7 & -0.7 & $-1.5^{*}$ \\
\hline
\end{tabular}

* Th as column contains the average $\Delta R_{y}$ and $\Delta R_{z}$ for all models, except GGCM. 

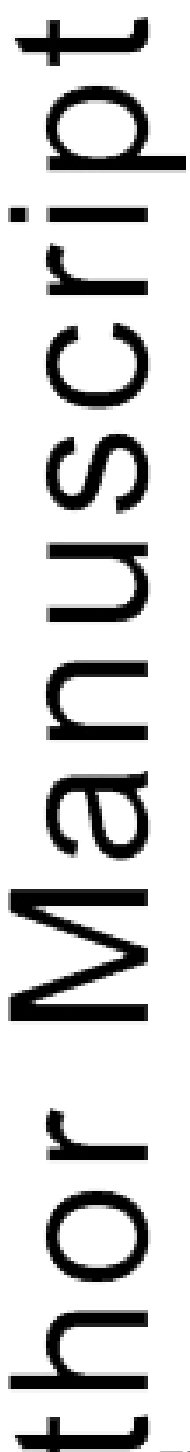

Figure 1. Electric current density obtained by the SWMF in the run $B z 0$ in the equatorial $(z=0)$ moon-meridional $(y=0)$ and terminator $(x=0)$ planes. Thick white lines indicate the boun $2 \mathrm{ry}$ between open and closed magnetic field lines determined by magnetic field line tracing. This boundary partly coincides with the maximum of electric current. We use the following solar wind conditions: $N=5 \mathrm{~cm}^{-3}, V_{x}=-400 \mathrm{~km} / \mathrm{s}, T=2 \times 10^{5} \mathrm{~K}, B_{y}=-B_{x}=3.5 \mathrm{nT}$ and $B_{z}=0$. The units in color bar are $\mathrm{nA} / \mathrm{m}^{2}$.

D R A F T

June 29, 2016, 9:56am

D R A F T 

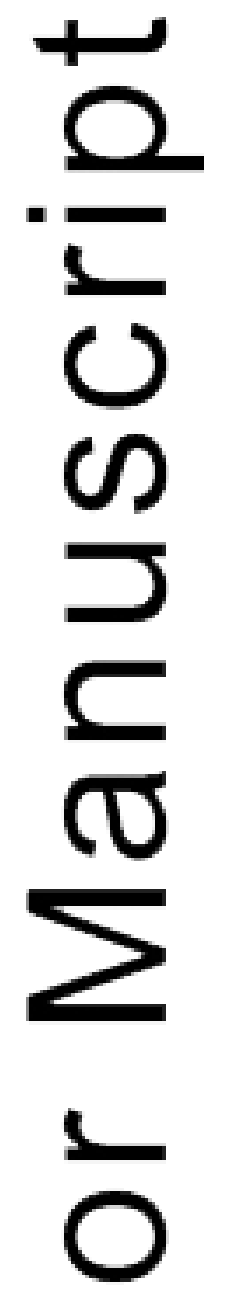

Figure positions in the equatorial and noon-meridional planes obtained by empirical and numerical MHD models: black solid [Shue et al., 1998], black dashed [Wang et al., 2013], blue (SWMF), green (LFM), and red lines (SPBU15 without the ring current). The solar wind contis ons are the same as those in Figure 1. 

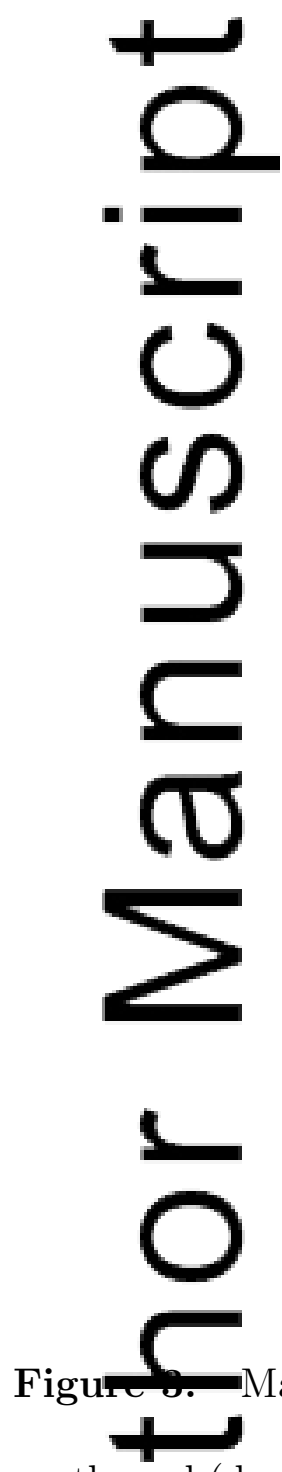

southward_dashed) IMF cases. Panel a: black [Shue et al., 1998], blue [Wang et al., 2013]; panel b: blue (SWMF), green (LFM), and red lines (SPBU15). Solar wind conditions are the following: $N=5 \mathrm{-an}, V_{x}=-400 \mathrm{~km} / \mathrm{s}, T=2 \times 10^{5} \mathrm{~K}, B_{y}=-B_{x}=3.5 \mathrm{nT}$ and $B_{z}= \pm 3 \mathrm{nT}$. 


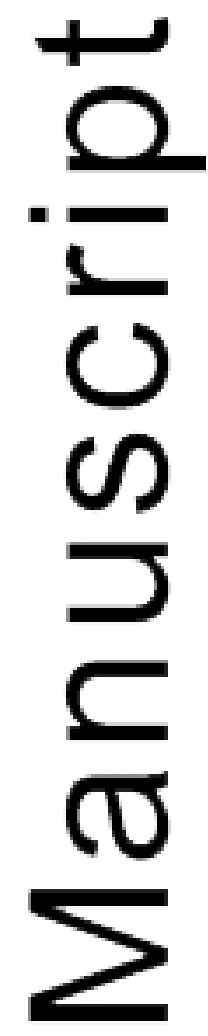

Figure Differences between the distances to the magnetopause for tilted and non-tilted dipoles $\left(R R\left(\Psi=15^{\circ}\right)-R\left(\Psi=0^{\circ}\right)\right)$ in the noon-meridional plane as a function of the latitude $\theta-\arctan (z / x)$. Solid black line corresponds to the W13 model, dashed black line to the B00 model red line to the L10 model, and blue line to the SWMF.

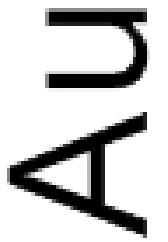




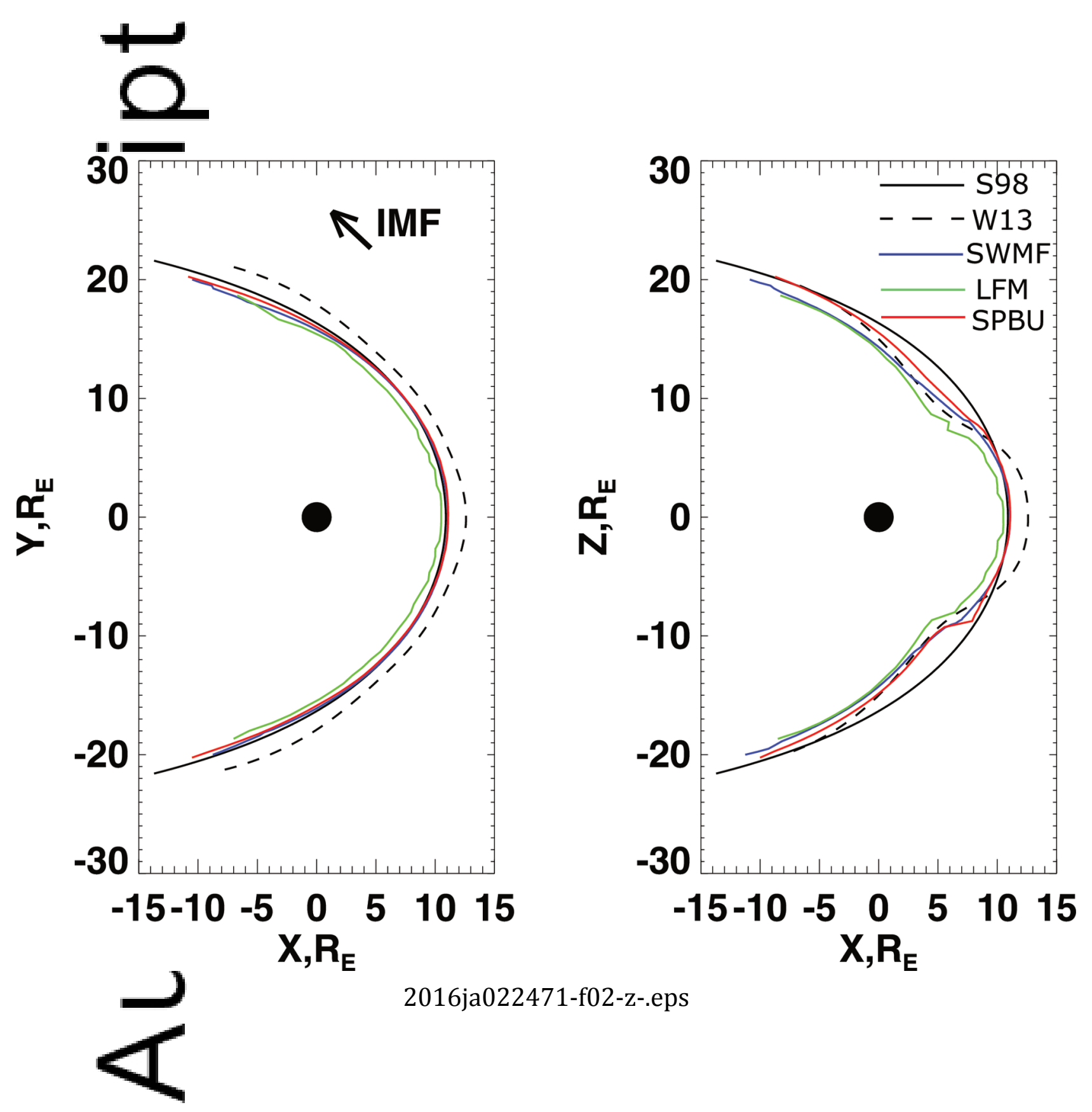

This article is protected by copyright. All rights reserved. 


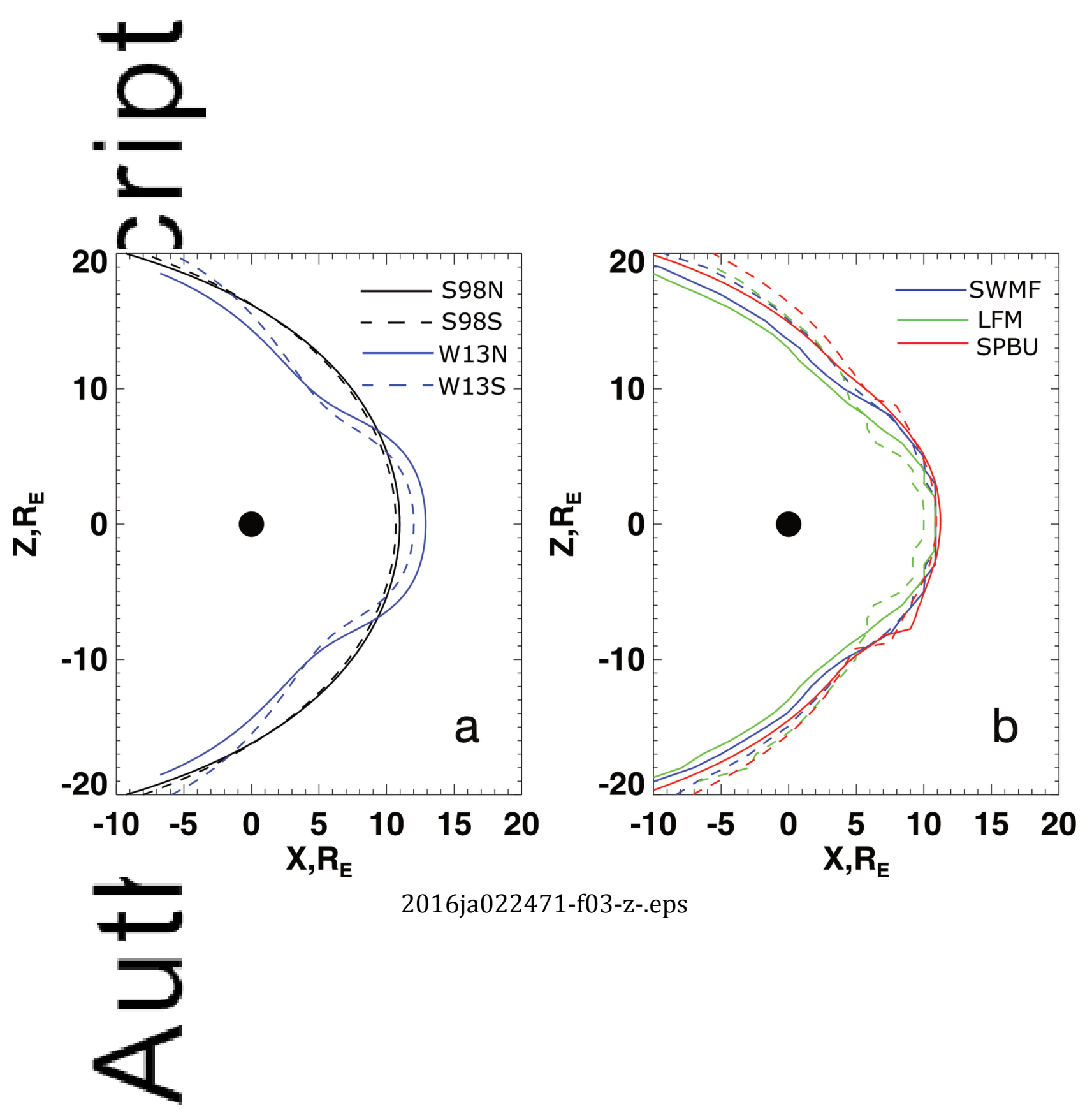

This article is protected by copyright. All rights reserved. 


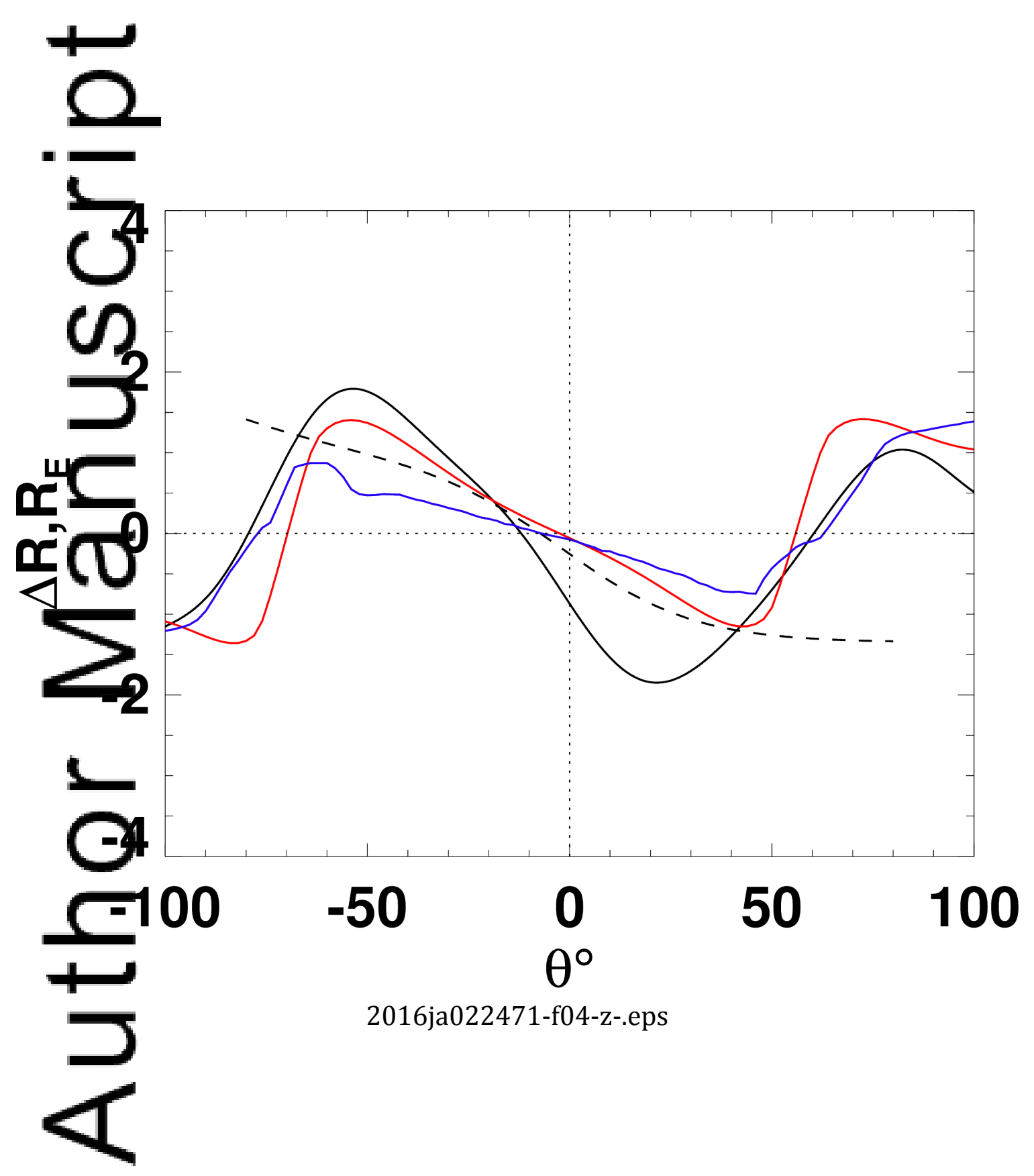

This article is protected by copyright. All rights reserved. 